DOI: https://doi.org/10.35699/2237-5864.2018.2436

\title{
MAPEAMENTO DE PRODUÇÕES BRASILEIRAS SOBRE O USO DA MODELAGEM MATEMÁTICA NO ENSINO DO CÁLCULO DIFERENCIAL E INTEGRAL
}

\author{
Jefferson Dantas de Oliveira ${ }^{1}$ \\ Zulma Elizabete de Freitas Madruga ${ }^{1}$
}

\section{RESUMO}

Este artigo apresenta resultados de uma pesquisa cujo objetivo foi investigar como a Modelagem Matemática (MM) se apresenta nas pesquisas acadêmicas que a utilizam para o ensino e a aprendizagem do Cálculo Diferencial e Integral (CDI). Como abordagem metodológica, utilizaram-se os procedimentos do Mapeamento na Pesquisa Educacional. Os dados foram constituídos a partir da seleção de dez pesquisas publicadas em três bancos de dados. Para a análise, estabeleceram-se inicialmente cinco categorias: a) referenciais teóricos da pesquisa; b) problemas investigados/interesses de pesquisa; c) metodologias utilizadas; d) principais resultados; e) perspectivas de continuidade do estudo. O estudo permitiu identificar a relevância da utilização de métodos como a MM no ensino e na aprendizagem da disciplina CDI. Verificou-se ainda que a utilização dessa metodologia permite que os estudantes sintamse mais interessados na aprendizagem dos conteúdos abordados na disciplina.

Palavras-chave: Ensino Superior. Modelagem Matemática. Cálculo Diferencial e Integral.

\footnotetext{
${ }^{1}$ Universidade Estadual de Santa Cruz (UESC), Ilhéus, BA, Brasil.
} 


\section{MAPPING OF BRAZILIAN PRODUCTIONS ABOUT USE OF MATHEMATICAL MODELING IN THE TEACHING OF DIFFERENTIAL AND INTEGRAL CALCULUS}

Jefferson Dantas de Oliveira

Zulma Elizabete de Freitas Madruga

\section{ABSTRACT}

This article presents results of a research about how Mathematical Modeling (MM) presents itself on academic researches that use MM on teaching and on the learning of Differential and Integral Calculus (ICD). As a methodological approach, Mapping in Educational Research procedures were used. The data were constituted from the selection of ten surveys published in three databases. For the analysis, five categories were initially established: a) theoretical references of the research; b) investigated problems/research interests; c) methodologies used; d) main results; and e) perspectives of continuity of the study. The study allowed us to identify the relevance of the use of methodological procedures such as $\mathrm{MM}$ in the teaching and learning of the CDI discipline. It was also verified that the use of this methodology allows the students to feel more interested in the learning of this contents covered on discipline.

Keywords: Higher education. Mathematical Modeling. Differential and Integral Calculus. 


\section{CONSIDERAÇÕES INICIAIS}

Um dos grandes desafios da Educação Matemática atualmente é buscar caminhos para o ensino da disciplina de forma a atrair a atenção e o gosto dos estudantes. Uma tarefa complexa, devido às dificuldades apresentadas por grande parte dos estudantes em relação a determinados conteúdos, dificuldades que geram falta de interesse pela Matemática.

Segundo Bassanezi (2010), o gosto pela Matemática se desenvolve com maior facilidade com a busca por estímulos que venham do mundo externo, ou seja, com a utilização em sala de aula de situações do cotidiano dos estudantes, desenvolvendo problemas que tenham como finalidade ensinar e estimular a criatividade dos alunos.

Por outro lado, o Cálculo Diferencial e Integral (CDI) não é uma disciplina que geralmente desperte o interesse dos estudantes. Segundo Guedin (2004), o CDI é muito diferente de outras disciplinas estudadas no Ensino Superior uma vez que os estudantes matriculados não tiveram contato anterior com os conceitos e as técnicas abordados. Portanto, é natural encontrarem dificuldades na compreensão de teoremas, propriedades e cálculos desenvolvidos na disciplina.

Nesta perspectiva, esta pesquisa investiga como a Modelagem Matemática (MM) se apresenta nas pesquisas acadêmicas que a utilizam para o ensino e a aprendizagem do CDI. Para tanto, apresentam-se a seguir um breve resgate teórico sobre o ensino do CDI e da MM, os procedimentos metodológicos utilizados nesta pesquisa, os resultados das pesquisas analisadas e as considerações finais deste estudo.

\section{ENSINO DE CÁLCULO E MODELAGEM MATEMÁTICA}

A Matemática, em qualquer nível de escolaridade, geralmente apresenta-se como uma prática que contempla o modelo "teoria-exemplo-exercício". Devido a essa forma de ensino, possivelmente alguns estudantes não conseguem compreender o conteúdo abordado, desenvolvendo dificuldades no estudo da disciplina. Essas dificuldades apresentadas pelos estudantes que ingressaram no Ensino Superior se refletem na aprendizagem dos conteúdos ministrados, principalmente no que diz respeito ao Cálculo, como afirma Guedin (2004). 
O CDI é uma disciplina que geralmente apresenta certo grau de dificuldade para os estudantes, principalmente devido ao fato de estes apresentarem lacunas quanto aos conteúdos básicos necessários para a compreensão do assunto. Segundo Gomes (2012), ao ingressarem na disciplina de CDI, os alunos se deparam com conteúdos complexos, que exigem um nível de abstração maior, algo que não foi devidamente trabalhado na Educação Básica.

Além das dificuldades encontradas nos conteúdos do Cálculo, Santos e Matos (2012) afirmam que os estudantes enfrentam dificuldades na metodologia utilizada pelo professor. Alguns professores que ministram a disciplina de CDI têm formação em diversas áreas, tais como Matemática, Física, Química, Ciências da Computação, Engenharias, entre outras. É fato que o professor de CDI conduzirá a disciplina de acordo com a sua área de atuação. Por exemplo, os professores de Matemática conduzem a disciplina pelo lado teórico, enquanto os professores da Engenharia preferem conduzir a disciplina pelo lado prático (WROBEL, et.al, 2013).

Acerca da metodologia de ensino, Brito e Cardoso (1997) salientam que os professores devem se atentar para abordar o conteúdo de uma forma na qual o estudante possa compreendê-lo, evitando omitir informações que possam ser importantes para o entendimento do assunto pelo aluno. Rezende (2003) ressalta que as dificuldades encontradas no ensino do CDI podem ser amenizadas de acordo com a metodologia aplicada pelo professor. Algumas dessas metodologias estimulam o aspecto cognitivo do estudante, de forma a auxiliá-lo a construir o seu aprendizado, acompanhado pelo professor. Um exemplo de metodologia que pode auxiliar no processo de ensino do CDI é a MM.

Conforme mostra Bassanezi (2010), a arte de buscar situações do cotidiano e adaptá-las nas aulas de Matemática é denominada MM. Diversos autores apresentam definições de MM, como Blum e Niss (1991, p. 40), “[...] o processo de modelagem é a prática de obter situações problema do cotidiano e relacioná-lo com a Matemática". Na mesma linha de pensamento, Bassanezi (2010, p. 16) afirma que "A Modelagem Matemática consiste na arte de transformar problemas da realidade em problemas matemáticos e resolvê-los interpretando suas soluções na linguagem do mundo real".

Madruga e Biembengut (2016, p. 28) apresentam outra definição:

Modelagem é o processo envolvido na feitura de um modelo. Modelo que pode auxiliar as pessoas a compreender dados, informações, a estimular novas ideias e a prover de uma visão estruturada e global que 
inclui relações abstratas de algum fenômeno, ente, ou um processo. O modelo capacita a pessoa a observar e refletir sobre fenômenos complexos, e ainda, a comunicar as ideias a outras pessoas.

Borba e Bizelli (1999) afirmam que a MM é uma metodologia que pode ser trabalhada em diversas áreas. A MM idealizada por Bassanezi (2010), Blum e Niss (1991), Madruga e Biembengut (2016) e Borba e Bizelli (1999) consiste em propostas metodológicas que podem ser utilizadas em qualquer área de ensino. Elas proporcionam a oportunidade de trabalhar com os conteúdos numa abordagem que utiliza situações do cotidiano dos estudantes, além de retomar essas situações na sala de aula, com vistas ao ensino e à aprendizagem.

\section{PROCEDIMENTOS METODOLÓGICOS}

Esta pesquisa é de cunho qualitativo (ALVES-MAZOTTI, 1998; BOGDAN; BIKLEN, 1994). Para tanto, utiliza-se os princípios do mapeamento da pesquisa educacional na perspectiva de Biembengut (2008). Para a autora, mapeamento é

um conjunto de ações que começa com a identificação dos entes ou dados envolvidos com o problema a ser pesquisado, para, a seguir, levantar, classificar e organizar tais dados de forma a tornarem mais aparentes as questões a serem avaliadas, reconhecer padrões, evidências, traços comuns ou peculiares, ou ainda características indicadoras de relações genéricas, tendo como referência o espaço geográfico, o tempo, a história, a cultura, os valores, as crenças e as ideias dos entes envolvidos (BIEMBENGUT, 2008, p. 74).

As pesquisas encontradas no desenvolvimento deste mapeamento foram obtidas por meio de buscas em três bases de dados específicas: 1) portal da Coordenação de Aperfeiçoamento de Pessoal de Nível Superior (CAPES); 2) revistas eletrônicas; e 3) Google Acadêmico. No portal da CAPES, foi realizada uma busca com as palavras-chave "modelagem matemática" e "cálculo diferencial e integral", e foi encontrado um total de 984.121 pesquisas. Em seguida utilizaramse os seguintes filtros (mostrados aqui com seus respectivos resultados entre parênteses): período de 2002 a 2017 (565.237); ciências exatas e da natureza (51.048); matemática e matemática aplicada (5.072); matemática, matemática computacional e modelagem (1.147). Após a filtragem, foi realizada uma leitura do título e das palavras-chave dos 1.147 trabalhos encontrados, com a finalidade de selecionar apenas as pesquisas que relacionassem a MM 
com o CDI. Esse processo reduziu o número de textos significativamente, totalizando 20 trabalhos.

As revistas eletrônicas encontradas, que foram inicialmente pesquisadas na plataforma Sucupira, ${ }^{2}$ totalizaram 366 periódicos, os quais possuem Qualis A1, A2 e B1. Desses, foram selecionadas apenas aqueles que tratam de Educação ou Educação Matemática, o que resultou na análise de 60 revistas, sendo 15 (Qualis A1), 20 (Qualis A2) e 25 (Qualis B1). Em cada uma dessas revistas, fez-se uma busca, na própria base de dados do periódico, por artigos que continham os termos "Modelagem Matemática" e "Cálculo Diferencial e Integral". Como resultado, foram encontradas apenas 5 pesquisas.

A busca no Google Acadêmico foi realizada com os termos "Modelagem Matemática" e "Cálculo Diferencial e Integral". Dedicou-se o foco às primeiras vinte páginas dos resultados, nas quais um total de 200 trabalhos relacionados foram encontrados. Ao selecionar apenas os trabalhos que relacionam os dois parâmetros e que foram produzidos nos últimos 15 anos, tem-se os resultados expressos no Quadro 1:

Quadro 1 - Resultado das buscas nas bases de dados

\begin{tabular}{ll}
\hline & “Modelagem Matemática” e “Cálculo Diferencial e Integral” \\
\hline Base de dados & Quantidade \\
CAPES & 20 \\
Revistas eletrônicas & 5 \\
Google Acadêmico & 15 \\
Total de produções encontradas & $\mathbf{4 0}$ \\
\hline
\end{tabular}

Fonte: elaborado pelos autores, 2018.

Após a leitura dos resumos de cada trabalho, foram selecionadas dez pesquisas para este estudo, as quais se referem especificamente ao objetivo de utilizar a MM com a finalidade de trabalhar os conteúdos do CDI. As referências dos trabalhos selecionados estão expressas no Quadro 2, em ordem aleatória, conforme os textos foram sendo encontrados.

\footnotetext{
${ }^{2}$ Banco de dados da CAPES que exibe revistas acadêmicas de todas as áreas do conhecimento.
} 
Quadro 2 - Apresentação das pesquisas selecionadas

BARROSO, N. M. C. et al. Modelagem de Conceitos e Processos Matemáticos por Redes de

A1 Petri Coloridas: o caso da integrabilidade de funções reais. Bolema, Rio Claro, v. 27, n. 45, p. 75-95, abr. 2013.

A2

ARAÚJO, J. L. Situações Reais e Computadores: os convidados são igualmente bem-vindos? Bolema, Rio Claro, v. 16, n. 19, maio 2003.

TREVISAN, E. P. Sólidos de revolução e o teorema de Pappus-Guldin: uma experiência em

A3 uma turma de cálculo de várias variáveis. Educação Matemática em Revista, Brasília, v. 22, n. 54, p. 106-115, abr./jun. 2017.

SILVA, K. A. P. Tarefas que Emergem em Atividades de Modelagem Matemática em um Ambiente Educacional de Cálculo Diferencial e Integral. JIEEM, v. 10, n. 1, p. 23-40, 2017.

SILVA, C. A. Introdução ao conceito de Integral de funções polinomiais em um curso de Engenharia de Produção por meio de tarefas fundamentadas em princípios da Modelagem Matemática. 2013. Tese (Doutorado) - Pontifícia Universidade Católica de São Paulo. São Paulo, 2013.

SPINA, C. O. C. Modelagem matemática no processo ensino aprendizagem do Cálculo Diferencial e Integral para o Ensino Médio. 2002. 177 f. Dissertação (Mestrado em Educação Matemática) - Instituto de Geociências e Ciências Exatas, Campus de Rio Claro. Rio Claro: Universidade Estadual Paulista, 2002.

FERRUZI, E. C. A Modelagem Matemática como estratégia de ensino e aprendizagem do Cálculo Diferencial e Integral nos Cursos Superiores de Tecnologia. 2003. 161 f. Dissertação (Mestrado em Engenharia de Produção) - Centro Tecnológico. Florianópolis: Universidade Federal de Santa Catarina, 2003.

ALMEIDA, L. M. W; FATORI, L. C; SOUZA, L. G. S. S. Ensino de Cálculo: uma abordagem usando A8 Modelagem Matemática. Revista Ciência e Tecnologia, [s. I.], v. 10, n. 16, jan. 2010. ISSN 2236-6733.

ARAÚJO, J. L. Cálculo, Tecnologias e Modelagem Matemática: As discussões dos alunos. A9 2002. 180 f. Tese (Doutorado em Educação Matemática) - Instituto de Geociências e Ciências Exatas Campus de Rio Claro. Rio Claro: Universidade Estadual Paulista, 2002.

BELTRÃO, M. E. P; IGLIORI, S. B. C. Modelagem Matemática e Aplicações: Abordagens Para o Ensino de Funções. Educação Matemática Pesquisa, São Paulo, v. 12, n. 1, p.17-42, 2010.

Fonte: elaborado pelos autores, 2018.

Após a seleção e o estudo das pesquisas encontrados, realizou-se a análise dos trabalhos de acordo com os procedimentos do mapeamento (BIEMBENGUT, 2008).

Para entender como as pesquisas utilizaram a MM no ensino do CDI, foram estabelecidos cinco critérios de análise para o estudo, definidos previamente como: a) referenciais teóricos da pesquisa; b) problemas investigados/interesses de pesquisa; c) metodologias utilizadas; d) 
principais resultados das pesquisas; e e) perspectivas de continuidade do estudo. Os principais pontos emergentes desta análise são descritos a seguir.

\section{RESULTADOS E DISCUSSÕES}

Nesta etapa da pesquisa, congregam-se os resultados obtidos em cada artigo com a produção teórica pertinente a cada categoria.

\section{a) O Referencial Teórico de MM nas Pesquisas}

A MM é uma área de pesquisa em processo de expansão, portanto há diversos pesquisadores que desenvolvem trabalhos com este enfoque. Os autores encontrados nos trabalhos foram:

- Bassanezi (2010): ${ }^{3}$ apresenta a MM como a arte de usar situações do cotidiano dos estudantes para ensinar Matemática. Nessa concepção encontraram-se os trabalhos: A4, A5, A6, A7, A8 e A9.

- Borba e Bizelli (1999): apresentam a MM como o processo no qual os alunos escolhem o conteúdo a ser trabalhado, e o professor auxilia no processo da construção do conhecimento. Nessa concepção encontraram-se os trabalhos: A1, A2, A3, A5, A6, A9, A10.

- Biembengut (2007): apresenta a MM como construção de um modelo no processo de ensino e aprendizagem, permitindo ao aluno aprender Matemática e outras disciplinas de forma conjunta. Nessa concepção encontraram-se os trabalhos: A5, A6, A7, A8, A9, A10.

- Blum e Niss (1991): apresentam a MM como o processo e a prática de obter situações-problema do cotidiano e relacioná-lo com a Matemática. Nessa concepção encontraram-se os trabalhos: A2 e A9.

Nos trabalhos coletados, a intenção foi identificar como os estudos de MM foram analisados teoricamente pelas pesquisas. Na análise verificou-se que essas pesquisas utilizam a modelagem

\footnotetext{
${ }^{3}$ Em algumas das pesquisas analisadas, os autores trazem referência mais antiga da obra de Bassanezi, como as edições de 2002 e 2004. Por tratar-se do mesmo livro, apenas com edições e/ou reimpressões diferentes, optou-se por utilizar a mais atual.
} 
como fundamentação teórica e, geralmente, o recurso de sequências de atividades para justificar a apropriação da MM como aporte teórico - em vez de propor situações-problema retiradas do cotidiano, nas quais os estudantes possam apresentar modelos. Além disso, foi possível verificar que o foco principal nos aportes teóricos de MM foi pautado em Bassanezi (2010), complementado com uma revisão de literatura sobre o tema.

\section{b) Os Problemas Investigados/Interesses de Pesquisa}

Quanto às pesquisas selecionadas, todas apresentam uma inquietação ou uma motivação para investigar a respeito de como a MM pode auxiliar no processo de ensino do CDI.

Em alguns trabalhos foi investigada de que forma a tecnologia utilizada por meio de ambientes computacionais pode auxiliar no processo de modelagem de situações para o ensino do CDI. Nessa perspectiva, encontram-se as pesquisas A2, A3 e A9. Vale salientar que os estudos se encaixam na mesma perspectiva, porém não têm os mesmos problemas e os mesmos objetivos. Pode-se observar essas diferenças nas pesquisas $\mathrm{A} 2$, em que a questão é "Existe um tratamento com iguais condições para a situação real e para o computador em um contexto de ensino e aprendizagem de matemática que considere modelagem e tecnologias?", e A9, cuja questão é "De que forma os alunos, por meio da MM, aprendem Cálculo em um ambiente computacional?". Observa-se que os dois estudos têm como tema o recurso computacional, porém com propósitos diferentes.

Outras pesquisas utilizam a MM como uma metodologia alternativa para que seja possível realizar o ensino do CDI, distanciando-se das aulas tradicionais. Nessa perspectiva encontramse as pesquisas: $A 1, A 6, A 7$ e A8. Observa-se que, na pesquisa $A 1$, o autor utiliza a Rede de Petros com a finalidade de ensinar a integrabilidade de funções. Já no estudo desenvolvido na A6, o autor se preocupa em investigar de que forma a interdisciplinaridade, por meio da MM, pode auxiliar os alunos na compreensão de conteúdos do CDI.

Nas pesquisas A4 e A10, foi identificado que os autores utilizam-se dos recursos de tarefas, por meio de sequências de atividades, para facilitar a compreensão de conteúdos do CDI. Vale salientar que outras pesquisas também utilizaram esse recurso, porém adotaram outras metodologias complementares. Por outro lado, a perspectiva de identificar as dificuldades apresentadas pelos alunos numa turma de CDI mediante processo de modelagem foi abordada na pesquisa $\mathrm{A} 5$. 
Contudo, em todas as pesquisas buscam-se analisar as reflexões, impressões e ações de alunos e professores sobre a MM no ensino do CDI, apresentando, assim, suas concepções e abordagens.

\section{c) Metodologia Utilizada nas Pesquisas em MM}

Observou-se que na maioria das pesquisas os autores assumem a abordagem qualitativa. Esse tipo de pesquisa é comum entre investigações no âmbito da Educação Matemática. Conforme Bogdan e Biklen (1994), a pesquisa qualitativa está relacionada ao levantamento de dados, compreensão e interpretação de comportamentos de um grupo. Esse tipo de pesquisa não prioriza resultados numéricos, mas o aprofundamento da compreensão de um grupo social, uma organização, entre outros.

Nas pesquisas selecionadas, as de cunho qualitativo são: A2, A3, A5, A6, A8 e A9. Quanto ao tipo de pesquisa, o trabalho A6, por exemplo, apresenta uma pesquisa-ação. Segundo Fiorentini e Lorenzato (2009), a pesquisa-ação se caracteriza pela necessidade do pesquisador de entranhar-se no processo de pesquisa e estar junto aos sujeitos que realizam a atividade para proporcionar ganhos no processo de ensino e aprendizagem do CDI. A pesquisa-ação aparece nos trabalhos $A 3, A 4, A 5, A 6, A 7$ e $A 10$.

O artigo A1 apresenta um estudo de caso que, segundo Ponte (1992), é uma investigação detalhada de algum contexto, ou seja, o investigador insere-se na pesquisa com o intuito de entender todas as causas de um problema. No caso do artigo A1, os autores utilizam a MM para estudar especificamente o caso de integrabilidade de funções.

Em relação aos sujeitos de pesquisa, as turmas em que foram aplicadas as intervenções eram geralmente de cursos de ciências exatas e tecnológicas. As principais turmas eram do curso de Licenciatura em Matemática, Licenciatura em Química e cursos de Engenharia. Os estudantes foram convidados a participar das intervenções assinando um termo de livre consentimento para o uso dos dados coletados.

Como instrumentos de coleta de dados, foram realizadas gravações de áudios e/ou vídeos das práticas de modelagem, criados relatórios e/ou produzidos materiais pelos estudantes, bem como examinados registros escritos produzidos pelos alunos: A1, A2, A3, A4, A5, A6, A9 
e A10. Em seguida, foram analisados os principais resultados encontrados em cada pesquisa, relatados pelos autores.

\section{d) Principais Resultados das Pesquisas em MM}

A análise dos trabalhos selecionados, em geral, apresenta uma diversificação de contribuições que a MM proporciona aos envolvidos no processo de ensino. De uma forma sintética, conforme o resultado apresentado pelas pesquisas analisadas, o uso da MM no processo de ensino do CDI facilitou a aprendizagem dos estudantes. A seguir, apresentam-se as principais considerações dos autores.

Em A1, as autoras afirmam que o uso das Redes de Petros, fundamentadas em teorias matemáticas, pode orientar o professor tanto na concepção de suas sequências de ensino quanto na escolha de atividades que conduzam o aluno a alcançar um nível maior de generalidade. $O$ intuito é o de contribuir, de maneira mais geral, com uma sistemática que auxilie o professor na organização de conteúdos complexos, tais como os conceitos de cálculo, interligando-os de forma matematicamente coerente e tornando-os mais acessíveis ao professor.

De acordo com A2, a autora conclui que a MM, com a finalidade do ensino do CDI, pode abrir possibilidades para que situações reais entrem na escola, com o objetivo de proporcionar a formação de cidadãos críticos. Certamente, a consideração de situações reais não garante, necessariamente, que o foco da formação seja a cidadania crítica, mas esta pode ser uma possibilidade proporcionada pela MM, que pode, também, promover um repensar sobre o papel dos computadores na escola.

Conforme A3, o autor explana que o desenvolvimento da intervenção com a MM proporcionou aos estudantes momentos para discussão de vários conceitos de outras áreas e da própria Matemática, como: centro de massa, área, volume e comprimento, função, matriz de rotação, parametrização, entre outros.

Nas pesquisas A4 e A6, os autores descrevem que os alunos fizeram uso de conhecimentos relativos a diferentes tipos de função com os quais tiveram contato quando utilizaram 
softwares como o Curve Expert. ${ }^{4}$ Calcularam limites e derivadas para deduzir um modelo matemático ou mesmo complementaram a situação em estudo. Dessa forma, pode-se concluir que a investigação de atividades de MM, desenvolvidas nas disciplinas de CDI, possibilita o envolvimento dos alunos com conteúdos matemáticos atrelados às situações próprias do contexto em que os estudantes estão inseridos, bem como o uso da tecnologia. No caso de A6, diferentes tarefas emergem com o objetivo de atingir uma meta que consiste no desenvolvimento da atividade de modelagem.

Em A5, o autor apresenta atividades de MM com o objetivo de verificar se as tarefas produziram significados. Posteriormente, os estudantes relataram sua aprendizagem. O intuito não foi o de realizar modelagem, segundo a visão de Bassanezi, mas o de buscar inserir os princípios dessa teoria em uma sequência de tarefas, a fim de levantar aspectos do processo de ensino. A escolha do tema, a interação e a Matemática envolvida, a construção de um modelo e a sua validação foram contempladas na oficina.

Conforme A7, a autora expõe que a Matemática pode oferecer modelos que influenciam o comportamento e que o uso da MM no ensino pode possibilitar que o CDI seja orientado à resolução de problemas, levando ao aprendizado do conteúdo de forma natural, de acordo com a demanda.

Segundo A8, as autoras apresentam uma dualidade no uso da MM no ensino do CDI e afirmam que: por um lado, pensar em atividades de Cálculo como algo que envolve "ideias" e "tem sentido" para os alunos parece ser fator importante para a aprendizagem, o que pode contribuir para amenizar os problemas percebidos em relação ao ensino e à aprendizagem da disciplina. Por outro lado, a atribuição de sentido e o desenvolvimento de ideias podem se dar a partir de problematizações introduzidas nas aulas.

Nos trabalhos A9 e A10 os estudantes apresentaram dificuldades na busca por situações do cotidiano quando foram solicitados. Para contornar as situações de obstáculos apresentados nas pesquisas A9 e A10, as autoras sugeriram aos alunos situações do cotidiano para que eles, por unanimidade, pudessem trabalhar em uma situação ideal.

\footnotetext{
${ }^{4}$ Software para plotagem de gráficos.
} 
Em A7, a autora salientou que as atividades de MM geram a habilidade de "aprender a aprender", que constitui a base para a construção de conhecimentos, pois, devido à situação proposta a ser trabalhada pelos estudantes, eles aprendem com os próprios erros e, dessa forma, há uma aprendizagem significativa por parte deles.

Em suma, as pesquisas selecionadas apresentam, em suas considerações, que a MM para o ensino e a aprendizagem de cálculo auxilia o aluno na forma que este pensa e aprende o conteúdo. Isso facilita a aprendizagem da disciplina, além de atrair a atenção dos estudantes, porém, houve entraves em algumas pesquisas no que diz respeito à escolha do tema a ser trabalhado, nas quais alguns estudantes ficaram receosos em apresentar uma situação para o desenvolvimento de um modelo matemático. Em seguida, apresentam-se as perspectivas de continuidade relatadas em algumas pesquisas.

\section{e) Perspectivas de Continuidade}

Em uma pesquisa acadêmica, a indicativa de continuidade supõe que o tema ainda pode ser explorado pelo autor ou por outros pesquisadores, tanto na mesma temática como por meio de outras vertentes. O presente mapeamento analisado mostrou que entre dez pesquisas relacionadas, apenas quatro apresentam perspectivas de continuidade. Os trabalhos $A 1, A 2$, A3, A7, A8 e A10 não as apresentaram.

Em A4, a autora sugere que sejam desenvolvidas novas atividades de pesquisas nas quais haja possibilidades de se trabalhar com outros objetos matemáticos. Nessa pesquisa, a autora utilizou um software em uma turma de CDI da Licenciatura em Química, a fim de modelar situações apresentadas pelos estudantes, e sugere que seja abordada a mesma situação proposta em outro curso e/ou com softwares diferenciados. Em A5, o autor desenvolve sua pesquisa em um curso de Engenharia, sugerindo que há possibilidades de se trabalhar com outras áreas do conhecimento, tais como a Física e a Química, entre outras. Em A6, a autora desenvolve um trabalho sobre a colmeia de abelhas, trabalhando com figuras geométricas de seis lados - hexágonos -, e apresenta a possibilidade de se trabalhar com outras figuras geométricas, tais como triângulos, retângulos, quadrados, entre outras. Em A9, a autora, ao se deparar com obstáculos na discussão do tema de modelagem a ser trabalhado, sugere em suas considerações que haja outras práticas de modelagem a serem utilizadas por outros pesquisadores. 
Dessa forma, as considerações dos trabalhos acima mencionados reforçam a ideia de que alguns estudos geralmente não são conclusivos, possibilitando novas investigações ou interpretações de outros pesquisadores e cenários com a mesma perspectiva de pesquisa. A seguir, apresentam-se algumas considerações finais sobre este estudo.

\section{CONSIDERAÇÕES FINAIS}

Esta pesquisa teve como objetivo investigar como a Modelagem Matemática (MM) se apresenta nos trabalhos acadêmicos que a utilizam para o ensino e a aprendizagem do Cálculo Diferencial e Integral (CDI). Para tanto, sob a ótica do Mapeamento na Pesquisa Educacional (BIEMBENGUT, 2008), foram selecionados e analisados dez trabalhos encontrados nos bancos de dados da CAPES, Google Acadêmico e Revistas de Qualis A1, A2 e B1.

As pesquisas selecionadas apresentam diversos autores que discorrem sobre o tema nas amplas visões da Modelagem, entre eles os principais nomes da MM no Brasil: Bassanezi (2010) e Biembengut (2007). Esses autores abordam a modelagem como processo no qual os estudantes, por meio de uma situação do cotidiano, desenvolvem um modelo matemático de situações propostas, tornando assim esses alunos responsáveis pelo próprio processo de aprendizagem, ao passo que o professor assume o papel de mediador do conhecimento.

Nas pesquisas selecionadas, os participantes eram estudantes dos cursos de Licenciatura em Matemática, Química e Engenharia. No geral, foi a primeira participação desses estudantes em uma pesquisa de modelagem, e as ideias norteadoras dos modelos em grande parte foram motivadas pelos participantes.

Na análise desses estudos observou-se que: 1) o uso da MM em uma aula do CDI permite que os estudantes interajam mais com o professor, pois se sentem parte do processo de aprendizagem; 2) em uma aula na disciplina de CDI, a MM funciona como uma proposta metodológica que é relevante para o processo de ensino e aprendizagem; 3) verificou-se ainda que a utilização dessa metodologia permite aos estudantes sentirem-se mais interessados no processo de aprendizagem do CDI, adquirindo o interesse pelo estudo; 4) nas pesquisas analisadas, a MM está geralmente associada com outras propostas metodológicas, ou ferramentas tecnológicas, com a finalidade de aprimorar o processo de ensino e aprendizagem; 5) houve trabalhos em que existiram algumas dificuldades em utilizar a MM na sala de aula; 
6) em algumas pesquisas foi observado que a MM serviu apenas como aporte teórico, sendo que, na prática, foi apresentada apenas uma sequência de atividades.

Por meio da análise dos trabalhos selecionados, percebeu-se que não há uma diversidade nas pesquisas que exploram o uso da MM para o ensino do $\mathrm{CDI}$, carecendo de mais pesquisas que abordem essa linha de investigação. Em relação às perspectivas de continuidade, destacamse as necessidades de compreender quais os principais conteúdos do CDI são abordados nos trabalhos que utilizam a MM e investigar quais as principais dificuldades dos estudantes em trazer uma situação do cotidiano para a sala de aula.

\section{REFERÊNCIAS}

ALVES-MAZZOTTI, Alda J. O método nas Ciências Sociais. In: GEWANDSNAJDER, F. O método nas Ciências Sociais: pesquisa quantitativa e qualitativa. São Paulo: Pioneira, 1998. cap. 4. p. 145-152.

BASSANEZI, Rodney C. Ensino-aprendizagem com Modelagem Matemática. 3. ed. São Paulo: Contexto, 2010.

BIEMBENGUT, Maria Salett. Modelagem Matemática \& Implicações no Ensino e Aprendizagem de Matemática. 3. ed. Blumenau: Edifurb, 2007.

BIEMBENGUT, Maria Salett. Mapeamento na Pesquisa Educacional. Rio de Janeiro: Editora Ciência Moderna, 2008.

BLUM, Werner; NISS, Mogens. Applied mathematical problem solving, modelling, applications, and links to other subjects - state, trends and issues in mathematics instruction. Educational Studies in Mathematics, Dordrecht, v. 22, n. 1, p. 37-68, 1991.

BOGDAN, Robert; BIKLEN, Sari. Investigação qualitativa em educação. Portugal: Porto Editora, 1994. p. 15-80.

BORBA, Marcelo de Carvalho; BIZELLI, Maria Helena S.S. O conhecimento matemático e o uso de softwares gráficos. Educação Matemática em Revista, n. 7, ano 6, p. 45, 1999.

BRITO, Arlete de J.; CARDOSO, Virgínia C. Uma abordagem histórico-pedagógica dos fundamentos do Cálculo Diferencial: reflexões metodológicas. Zetetiké, v. 5, n. 1, p. 129144, 1997. 
FIORENTINI, Dario; LORENZATO, Sergio. Investigação em educação matemática: percursos teóricos e metodológicos. 2. ed. Campinas: Autores Associados, 2009. 240 p.

GOMES, Eloiza. Ensino e aprendizagem de cálculo na engenharia: um mapeamento das publicações nos COBENGE. In: ENCONTRO BRASILEIRO DE ESTUDANTES DE PÓSGRADUAÇÃO EM EDUCAÇÃO MATEMÁTICA, 16., 2012, Canoas. Anais... Canoas: ULBRA, 2012.

GUEDIN, Juliana. Cálculo diferencial e integral: o ensino como uma abordagem histórica e suas contextualizações. 2004. Monografia (Especialização em Educação Matemática) - Universidade do Extremo Sul Catarinense. Criciúma, 2004.

MADRUGA, Zulma E. de F; BIEMBENGUT, Maria S. Modelagem e Aleg(o)rias: um enredo entre cultura e educação. Curitiba: Editora Appris, 2016.

REZENDE, Wanderley M. O ensino de Cálculo: dificuldades de natureza epistemológica. In: MACHADO, Nilson José; CUNHA, Marisa (Org.). Linguagem, Conhecimento, Ação ensaios de epistemologia e didática. São Paulo: Escrituras, 2003.

PONTE, João Pedro da. Concepções dos professores de Matemática e processos de formação. In: BROWN, M. et al. (Ed.). Educação e Matemática: Temas de investigação. Lisboa: IIE e Secção de Educação e Matemática da SPCE, 1992. p. 186-239.

SANTOS, Sílvia P. dos; MATOS, Marcia G. de O. O ensino de Cálculo I no curso de Licenciatura em Matemática: obstáculos na aprendizagem. Revista Eventos Pedagógicos, v. 3, n. 3, p. 458-473, ago./dez. 2012.

WROBEL, Julia S. et al. O mapa do ensino de Cálculo nos últimos 10 anos do COBENGE. In: CONGRESSO BRASILEIRO DE EDUCAÇÃO EM ENGENHARIA, 41., 2013, Gramado. Anais... Gramado, Rio Grande do Sul: UFRGS, 2013.

\section{Jefferson Dantas de Oliveira}

Mestre em Educação Matemática pela Universidade Estadual de Santa Cruz (UESC). Licenciado em Matemática pela UESC.

dantascpm2a@hotmail.com 


\section{Zulma Elizabete de Freitas Madruga}

Doutora em Educação em Ciências e Matemática pela Pontifícia Universidade Católica do Rio Grande do Sul (PUCRS). Docente do Programa de Pós-graduação em Educação Matemática da UESC.

betefreitas.m@gmail.com 